keln ist demnach wesentlich durch letztern Umstand mitbedingt, und nicht ansschliesslich auf die beständige Absonderung von Urin mnd Koth auch im scheintodten Zustand zurückzuführen.

6. bei längerem Winterschlaf findet eine auffallend reichliche Glykogenbildung in der Leber statt.

Ueber den Zusammenhang der einzelnen Erscheinungen werden ansführliche und genane quantitative Bestimmungen bald näheren Anfschluss bringen.

\title{
Berichtigungen.
}

Seite.

Band II.

303 Z. 23 v. 0. lies: Substanz st. Base.

\section{Band III.}

1 Z. 8 v. u. lies: wirksamen st. wichtigen.

4 Z. 12 v. o. lies: Barythydrat st. Barythhydrat.

4 Z. 14 v. o. lies: des wirksamen Bestandtheils st. der wirksamen Bestandtheile.

4 Z. 10 v. u. lies : zum grössten Theile st. im grössten Theile.

6 Z. 36 v. o. lies: ferneren st. strengeren.

7 Z. 5 v. u. lies : ertheilt st. erheilt.

8 Z. 6 v. o. lies: ein Filter st. einen Filter.

8 Z. 19 v. 0. lies: Pharm. German. Dasselbe st. Pharm. German., dasselbe.

12 Z. 11 v. u. lies: Ergotins st. Ergotin.

14 Z. 9 v. u. lies: des Pilzes im Mutterkorn ganz st. des Pilzes ganz.

$15 \mathrm{Z} .14$ v. u. lies: werde st. würde.

15 Z. 4 v. u. lies: raubt st. erlaubt.

17 Z. 17 v. o. lies: und der Niederschlag st. und.
Seite.

20 Z. 16 v. u. lies: $(1: 2-3)$ tritt st. $(1: 2-3)$.

21 Z. 17 v. 0. lies: 53,21 st. 53,22.

22 Z. 9 v. u. lies: alkoholischer st. alkalischer.

23 Z. 9 v. o. lies : Digitoneïn st. Digitoresin.

23 Z. 3. v. u. lies: Digitoresin st. Digitonein

25 Z. 11 v. u. lies: Krystallform st. Form der Krystalle.

$30 \mathrm{Z} .17 \mathrm{v}$. o. lies: sich stets st. stets.

$31 \mathrm{Z}$. 6 v. 1 . lies: Färbung an ${ }^{\circ}$ st. Färbung.

$32 \mathrm{Z}$. 2.1 v. u. lies: $\mathrm{x}\left(\mathrm{C}_{5} \mathrm{H}_{8} \mathrm{O}_{2}\right)$ st. $\left(\mathrm{C}_{5} \mathrm{H}_{3} \mathrm{O}_{2}\right)$.

33 Z. 15 v. u. lies: Schwefelsüure und Brom und in Bezug auf seine Spaltung st. Salzsäure und Brom, in Bezug auf seine feine Spaltung.

35 Z. 11 v. n. lies: Grm. st. Cgrm.

37 Z. 7 v. u. lies: derselben st. desselben.

40 Z. 5 v. o. lies: $\left(\mathrm{C}_{21} \mathrm{H}_{33} \mathrm{O}_{7}\right)$ st. $\left(\mathrm{C}_{21} \mathrm{H}_{82} \mathrm{O}_{7}\right)$.

$40 \mathrm{Z}$. 6 v. 0 . lies: 63,48 st. $63,63$.

43 Z. 12 v. u. lies: sehr st. so sehr.

$55 \mathrm{Z} .7$ v. u. lies: Dgm. st. Cgm. 